\title{
Sparse solutions to an underdetermined system of linear equations via penalized Huber loss
}

\author{
Can Kızılkale ${ }^{1} \cdot$ Mustafa Ç. Pınar ${ }^{2}$
}

Received: 11 October 2019 / Revised: 28 October 2020 / Accepted: 28 October 2020

(c) Springer Science+Business Media, LLC, part of Springer Nature 2020

\begin{abstract}
We investigate the computation of a sparse solution to an underdetermined system of linear equations using the Huber loss function as a proxy for the 1-norm and a quadratic error term à la Lasso. The approach is termed "penalized Huber loss". The results of the paper allow to calculate a sparse solution using a simple extrapolation formula under a sign constancy condition that can be removed if one works with extreme points. Conditions leading to sign constancy, as well as necessary and sufficient conditions for computation of a sparse solution by penalized Huber loss, and ties among different solutions are presented.
\end{abstract}

Keywords Sparse solution - Linear system of equations - Compressed sensing · Basis pursuit $\cdot$ Huber loss function $\cdot$ Convex quadratic splines $\cdot$ Linear programming $\cdot \ell_{1}$-norm $\cdot$ Quadratic perturbation $\cdot$ Strictly convex quadratic programming

\section{Introduction and Background}

The purpose of the present brief paper is to prove an extrapolation property to calculate (the) sparse solutions to an underdetermined linear system by approximating the Basis Pursuit (BP) model, and to explore ties between sparse solutions, the L1-norm solution and the Huber loss function (c.f. Huber 1981). The problem of interest is posed as:

C. Kizılkale: The research of this author was supported in part by the Applied Mathematics program of the DOE Office of Advanced Scientific Computing Research under Contract No. DE-AC02$05 \mathrm{CH} 11231$.

Mustafa Ç. Pınar

cpinar64@yahoo.com

1 Lawrence Berkeley National Laboratory, Berkeley, CA 94720, USA

2 Faculty of Engineering, Bilkent University, 06800 Ankara, Turkey 


$$
\min _{x}\left\{\|x\|_{0}: A x=b\right\}
$$

where $\|x\|_{0}$ counts the non-zero elements of the vector $x \in \mathbb{R}^{n}, A \in \mathbb{R}^{m \times n}(m<n)$ assumed to be a full rank matrix (with rank equal to $m$ ), and $b \in \mathbb{R}^{m}$. The problem has numerous applications in signal processing, compressed sensing, error decoding, image denoising and so on. Since it is NP-hard, one may seek to solve an approximate problem instead of (1). A popular choice is the Basis Pursuit method (Chen et al. 1998). The Basis Pursuit approach to sparse recovery (there is ample literature on the subject, which cannot be reviewed within this brief paper; see e.g., (Bryan and Leise 2013; Candès and Tao 2006; Donoho 2005, 2006), or (Elad 2010; Foucart and Rauhut 2013) for in-depth monographs on the problem of compressive sensing), and is based on the following problem referred to as [SL1]:

$$
\min _{x}\left\{\|x\|_{1}: A x=b\right\} .
$$

In addition to the commonly observed fact that the problem (2) usually gives a sparsest solution to the linear system in numerical calculation, and with high probability according to several theoretical results in literature, it was proved that (2) is the Lagrange bi-dual of (1); c.f., (Singaraju et al. 2012). An alternative view to duality for (1) can be found in Chrétien (2010).

Let $f^{*}$ denote the optimal value of (2) and $X_{*}$ denote its optimal solution set. The dual problem is

$$
\max _{\xi}\left\{b^{T} \xi:\left\|A^{T} \xi\right\|_{\infty} \leq 1\right\} .
$$

Denote the optimal set of the dual problem as $\Xi^{0}$. The notation $s^{0}$ refers to the sign vector with components the usual sign function denoted sgn in $\{0, \pm 1\}$ such that

$$
\operatorname{sgn}(t)=\left\{\begin{aligned}
0 & \text { if } t=0 \\
1 & \text { if } t>0 \\
-1 & \text { if } t<0
\end{aligned}\right.
$$

The diagonal matrix $W^{0}($.$) is obtained from s^{0}($.$) using W_{i i}^{0}=1-\left(s_{i}^{0}\right)^{2}$ for $i=1, \ldots, n$. Optimality conditions for (2) can be summarized in the following result (see e.g., Fuchs 2004) which is convenient for our purposes.

Proposition 1 Let $x \in \mathbb{R}^{n}$ with $s=s^{0}(x)$ and the associated $W=W^{0}(x)$. Then, $x$ solves (2) if and only if $A x=b$ and there exists $\lambda \in \mathbb{R}^{m}$ and $d \in \mathbb{R}^{n}$ such that $\|W d\|_{\infty} \leq 1$ and $A^{T} \lambda=W d+s$.

Clearly, if the minimizer $x$ is unique in (2) the matrix $\left(\begin{array}{c}A \\ W\end{array}\right)$ has full rank. An alternative form of optimality conditions known as complementary slackness conditions, which is at times more convenient (equivalent to the conditions of Proposition 1) can be given as follows. Let $\left(x^{*} ; \xi^{*}\right)$ be a primal-dual feasible solution pair to (2) and (3); they are an optimal pair if and only if 


$$
\max \left\{x_{i}^{*}, 0\right\}\left(1-\left[A^{T} \xi^{*}\right]_{i}\right)=0, \min \left\{x_{i}^{*}, 0\right\}\left(1+\left[A^{T} \xi^{*}\right]_{i}\right)=0, i=1, \ldots, n .
$$

Now, we define the Huber loss function depending on a tuning constant $\gamma>0$ :

$$
\rho(t)= \begin{cases}\frac{1}{2 \gamma} t^{2}, & \text { if }|t| \leq \gamma \\ |t|-\frac{\gamma}{2}, & \text { otherwise }\end{cases}
$$

The Huber function is an example of a convex quadratic spline (a piecewise quadratic); see e.g., (Chen et al. 2005) for background on convex quadratic splines. In a previous paper (Pinar 2019) the second author investigated necessary and sufficient conditions for exact recovery of an individual sparse vector using the Huber function instead of the $\mathrm{L} 1$ function, i.e., using the problem

$$
\min _{x}\left\{\sum_{i=1}^{n} \rho\left(x_{i}\right) \mid A x=A u\right\}
$$

where $u$ is some sparse real vector. The approach of the present paper is different since we shall consider the problem

$$
\min _{x}\left\{\frac{1}{2 \gamma}\|b-A x\|_{2}^{2}+\Phi_{\gamma}(x)\right\}
$$

where $\Phi_{\gamma}(x)=\sum_{i=1}^{n} \rho\left(x_{i}\right)$, as an approximation to (2) for smaller and smaller values of $\gamma$. We shall refer to the problem as penalized Huber Loss as the first term acts as a simple penalty function. Problems that are reminiscent of (5) were studied in the context of linear and quadratic programming in Madsen and Nielsen (1993), Madsen et al. (1994), Madsen et al. (1998). Reference (Fuchs 2004) is also close in spirit to the present paper in that it utilizes quadratic programming problem similar to ours for computing a sparse solution although neither Huber loss function nor extrapolation ideas are present in Fuchs (2004). Another reference related to the present is Selesnick (2017) where the Huber function is used within the context of a min-max concave penalty and saddle point computations for least squares regularization. In Lanza et al. (2019), Wang et al. $(2018,2019)$ the Huber loss function (and its generalization) are used as the basis of a minimax-concave penalty in the context of regularized least squares for sparse recovery in engineering applications. There is yet another stream of research that is in line with the theme of the present paper. The reference (Wen et al. 2010) studies the solution of the problem with the L1-Least Squares objective $\min _{x}\left\{\|b-A x\|_{2}^{2}+\mu\|x\|_{1}\right\}$, for a decreasing sequence of positive parameters $\mu$ using a two-phase algorithm embedded in a continuation framework. The two-phase algorithm has a first-order iterative shrinkage phase where non-zero components are identified, followed by the solution of smaller size quadratic optimization problem. In Aybat and Iyengar (2011), a first-order smoothed penalty algorithm is designed for solving the problem $\min _{x}\left\{\|b-A x\|_{2}+\mu\|x\|_{1}\right\}$, for a decreasing sequence of parameters $\mu$ as in Wen et al. (2010). However, the subproblems are solved using the Nesterov optimal algorithm for simple sets (Nesterov 2004, 2005). The update step in the Nesterov algorithms involves the computation of the gradient 
of a suitably smoothed version of the function $\|b-A x\|_{2}+\mu\|x\|_{1}$ and solving minimum norm problems of the form $\min \left\{c^{T} x+\frac{L}{2}\|x-z\|_{2}^{2}:\|x\|_{2} \leq \beta\right\}$. This is close in spirit to the theme of the present paper as the Huber loss function admits a similar variational representation: $\Phi_{\gamma}(x)=\min _{y}\left\{y^{T} x+\frac{\gamma}{2}\|y\|_{2}^{2}:\|x\|_{\infty} \leq 1\right\}$. In Aybat and Iyengar (2012), a first-order augmented lagrangian algorithm is proposed for basis pursuit. The algorithm is reported to correctly identify the support of a target signal in numerical trials without the need of any thresholding. A more recent set of references (Cheng and Dai 2018, 2019; Cheng et al. 2009, 2015; Cheng and Li 2018; Lee et al. 2014) explore similar themes. In Cheng and Dai (2018), a more general problem of the form $\min _{x}\left\{f(x)+\mu\|x\|_{1}\right\}$, where $f$ is continously differentiable, is tackled with a gradient-based active set identification algorithm. In Cheng et al. (2015), an algorithm framework for the more general problem of minimizing the sum $f(x)+\psi(x)$, where $f$ is smooth and $\psi$ is convex, but possibly non-smooth, is presented. At each step, the search direction of the algorithm is obtained by solving an optimization problem involving a quadratic term with diagonal Hessian coupled with a non-monotone line search technique. In Lee et al. (2014) a similar problem is studied and a Newton-type algorithm is proposed. An iterative shrinkage thresholding algorithm with active set identification properties along with a Newton-Conjugate Gradient scheme for L1 optimization is proposed in Cheng and Dai (2019) for the problem $\min _{x}\left\{f(x)+\mu\|x\|_{1}\right\}$. Similarly, a fast conjugate gradient algorithm for active set prediction and a preconditioned conjugate gradient algorithm are respectively proposed in [9] and Cheng and Li (2018), for L1-regularized estimation.

The problem (5) aims to achieve two goals at the same time by pushing $\gamma$ to zero: recover a solution to the system $A x=b$ in the limit while the Huber loss function collapses to the 1-norm. It may be thought that the method advocated here will cause numerical problems as one seems to have to push $\gamma$ to extremely small values to obtain an approximate result. However, the crux of the results of the paper is that one does not need to deal with very small values of $\gamma$ due to a convenient extrapolation result described in Sect. 2. A variant of the extrapolation property using extreme point minimizers is discussed in Sect. 3. Section 4 is devoted to further exploration of the ties between a sparse solution, the unique solution to problem (2), and the penalized Huber Loss problem (5).

\section{An extrapolation procedure}

The first (to be expected) result is that the solutions of (5) tend to the solutions of (2) as $\gamma$ tends to zero.

Proposition 2 The sequence of solutions $\left\{x^{\gamma}\right\}$ of (5) approaches $X_{*}$ as $\gamma$ tends to zero: $\forall \epsilon>0, \exists \gamma(\epsilon)$ such that for $\gamma \leq \gamma(\epsilon), x^{\gamma}$ solves (5) $\Longrightarrow$ $\operatorname{dist}\left(x^{\gamma}, X_{*}\right)=\min _{x \in X_{*}}\left\|x^{\gamma}-x\right\| \leq \epsilon$.

Proof Let $x_{0}$ be a solution to (2). For ease of notation, define $f_{\gamma}(x)=\frac{1}{2 \gamma}\|b-A x\|_{2}^{2}+\Phi_{\gamma}(x)$. We have for all $x \in \mathbb{R}^{n}$ : 


$$
0 \leq\|x\|_{1}-\Phi_{\gamma}(x) \leq n \frac{\gamma}{2}
$$

Let $x^{i}$ denote a minimizer of $f_{\gamma_{i}}$. Then one has

$$
\left\|x^{i}\right\|_{1} \leq\left\|x^{i}\right\|_{1}+\frac{1}{2 \gamma_{i}}\left\|b-A x^{i}\right\|_{2}^{2} \leq \Phi_{\gamma_{i}}\left(x^{i}\right)+n \frac{\gamma_{i}}{2}+\frac{1}{2 \gamma_{i}}\left\|b-A x^{i}\right\|_{2}^{2} .
$$

On the other hand we have that

$$
\Phi_{\gamma_{i}}\left(x^{i}\right)+\frac{1}{2 \gamma_{i}}\left\|b-A x^{i}\right\|_{2}^{2} \leq f^{*}
$$

because

$$
\Phi_{\gamma_{i}}\left(x^{i}\right)+\frac{1}{2 \gamma_{i}}\left\|b-A x^{i}\right\|_{2}^{2} \leq \Phi_{\gamma_{i}}\left(x_{0}\right)+\frac{1}{2 \gamma_{i}}\left\|b-A x_{0}\right\|_{2}^{2} \leq\left\|x_{0}\right\|_{1}=f^{*} .
$$

Using (8) in (7) we get

$$
\left\|x^{i}\right\|_{1} \leq f^{*}+n \frac{\gamma_{i}}{2}
$$

Now, let $x^{i} \rightarrow \bar{x}$, if necessary after passing to a subsequence. Then $\bar{x}$ must satisfy $A x=b$ since otherwise we have

$$
\lim _{i \rightarrow \infty, \gamma_{i} \searrow 0} \Phi_{\gamma_{i}}\left(x^{i}\right)+\frac{1}{2 \gamma_{i}}\left\|b-A x^{i}\right\|_{2}^{2}=\|\bar{x}\|_{1}+\lim _{i \rightarrow \infty} \frac{1}{2 \gamma_{i}}\left\|b-A x^{i}\right\|_{2}^{2}
$$

and

$$
\lim _{i \rightarrow \infty, \gamma_{i} \searrow 0} \frac{1}{2 \gamma_{i}}\left\|b-A x^{i}\right\|_{2}^{2}=+\infty
$$

This leads to a contradiction with (8). So $\bar{x}$ satisfies $A \bar{x}=b$. By (7) one has

$$
\|\bar{x}\|_{1}=\lim _{i \rightarrow \infty}\left\|x^{i}\right\|_{1} \leq f^{*} .
$$

Hence $\bar{x} \in X_{*}$.

While the above gives a convergence result in the limit, we shall not have to let $\gamma$ tend to zero. We shall instead devise a simple extrapolation procedure to get to a solution of (2) from a solution of (5) under a sign constancy condition.

The optimality conditions of (5) can be expressed in a compact notation as follows; define $s^{\gamma} \in\{0, \pm 1\}^{n}$ as follows:

$$
s_{i}^{\gamma}(t)=\left\{\begin{aligned}
0 & \text { if }|t| \leq \gamma \\
1 & \text { if } t>\gamma \\
-1 & \text { if } t<-\gamma
\end{aligned}\right.
$$


with the diagonal matrix $W^{\gamma}($.$) derived from s^{\gamma}($.$) using W_{i i}^{\gamma}=1-\left(s_{i}^{\gamma}\right)^{2}$ for $i=1, \ldots, n$. Then, the following result can be stated.

Lemma 1 Let $\bar{x} \in \mathbb{R}^{n}$. Then $\bar{x}$ solves (5) if and only if the following equation holds:

$$
\frac{1}{\gamma} W^{\gamma}(\bar{x}) \bar{x}+s^{\gamma}(\bar{x})-\frac{1}{\gamma} A^{T}(b-A \bar{x})=0 .
$$

We shall obtain an extrapolation procedure based on Lemma 1. Let us rewrite equation (11) for a minimizer $x^{\gamma}$ as

$$
\left(A^{T} A+W^{\gamma}\left(x^{\gamma}\right)\right) x^{\gamma}=A^{T} b-\gamma s^{\gamma}\left(x^{\gamma}\right) .
$$

Corollary 1 Let $x^{\gamma}$ solve (5) uniquely. Then $\left(\begin{array}{c}A \\ W^{\gamma}\left(x^{\gamma}\right)\end{array}\right)$ has full rank.

Proof If $\left(\begin{array}{c}A \\ W^{\gamma}\left(x^{\gamma}\right)\end{array}\right)$ does not have full rank, there exists $h \neq 0$ such that $\left(\begin{array}{c}A \\ W^{\gamma}\left(x^{\gamma}\right)\end{array}\right) h=0$, which implies that there exists $\delta>0$ such that $s^{\gamma}\left(x^{\gamma}+\delta h\right)=s^{\gamma}\left(x^{\gamma}\right)$.

Lemma 2 Let $x^{\gamma}$ solve (5). Then the system of linear equations

$$
\left(A^{T} A+W^{\gamma}\left(x^{\gamma}\right)\right) d=s^{\gamma}\left(x^{\gamma}\right)
$$

is consistent.

Proof By rearranging (12) we obtain

$$
-\frac{1}{\gamma}\left(A^{T} W^{\gamma}\left(x^{\gamma}\right)\right)\left(\begin{array}{c}
r^{\gamma} \\
x^{\gamma}
\end{array}\right)=s^{\gamma}\left(x^{\gamma}\right)
$$

where $r^{\gamma} \equiv A x^{\gamma}-b$. Then (13) is equivalent to

$$
\left(A^{T} W^{\gamma}\left(x^{\gamma}\right)\right)\left(\begin{array}{c}
A \\
W^{\gamma}\left(x^{\gamma}\right)
\end{array}\right) d=-\frac{1}{\gamma}\left(A^{T} W^{\gamma}\left(x^{\gamma}\right)\right)\left(\begin{array}{c}
r^{\gamma} \\
x^{\gamma}
\end{array}\right)
$$

which is consistent since it is the normal equations for the system

$$
\left(\begin{array}{c}
A \\
W^{\gamma}\left(x^{\gamma}\right)
\end{array}\right) h=\left(\begin{array}{l}
r^{\gamma} \\
x^{\gamma}
\end{array}\right)
$$

For ease of notation let $W=W^{\gamma}\left(x^{\gamma}\right)$ and $s=s^{\gamma}\left(x^{\gamma}\right)$. Let the SVD of $\left(\begin{array}{l}A \\ W\end{array}\right)=U \Sigma V^{T}$ where $U \in \mathbb{R}^{(m+n) \times(m+n)}, \Sigma \in \mathbb{R}^{(m+n) \times n}, V \in \mathbb{R}^{n \times n}$ with $U^{T} U=I_{(m+n)}$ 
and $V V^{T}=V^{T} V=I_{n}$. Then we have $A^{T} b=\sum_{j=1}^{n} \alpha_{j} v_{j}$ where $v_{j}$ s are columns of $V$, and $s=\sum_{j=1}^{n} \beta_{j} v_{j}$, for some scalars $\alpha_{j}$ s and $\beta_{j}$ s. More compactly, we have $A^{T} b-\gamma s=V \alpha-\gamma V \beta$.

Now, let us re-write (12) as

$$
V S^{2} V^{T} x^{\gamma}=V \alpha-\gamma V \beta
$$

where $S^{2}=\Sigma^{T} \Sigma$ is $n \times n$ diagonal matrix with diagonal entries $\sigma_{j}^{2}, j=1, \ldots, n\left(\sigma_{j}\right.$ are singular values of $\left(\begin{array}{c}A \\ W\end{array}\right), \alpha$ is the vector of $\alpha_{j}$ s, and $\beta$ is the vector with components $\beta_{j}$ s. If $\left(\begin{array}{c}A \\ W\end{array}\right)$ has full rank, then $S^{2}$ is invertible, i.e., $\sigma_{j}>0$ for all $j=1, \ldots, n$. Therefore, an expression for $x^{\gamma}$ is obtained as

$$
x^{\gamma}=\sum_{j=1}^{n} \frac{\alpha_{j}-\gamma \beta_{j}}{\sigma_{j}^{2}} v_{j} .
$$

Now, if $s^{\gamma}\left(x^{\gamma}\right)$ remains constant at some $s$ for $\gamma \in\left(0, \gamma^{*}\right]$, one has

$$
\lim _{\gamma \rightarrow 0} x^{\gamma}=\sum_{j=1}^{n} \frac{\alpha_{j}}{\sigma_{j}^{2}} v_{j} \equiv x^{\#}
$$

Now, consider the linear system of equations

$$
\left(A^{T} A+W\right) d=s
$$

for $\gamma \in\left(0, \gamma^{*}\right]$ (i.e., the range of $\gamma$ where the sign vector remains constant). By the analysis above and under the full rank assumption on $\left(\begin{array}{l}A \\ W\end{array}\right)$, one has

$$
d=\sum_{j=1}^{n} \frac{\beta_{j}}{\sigma_{j}^{2}} v_{j}
$$

Hence, using the expression (14) for $x^{\gamma}$ gives

$$
x^{\gamma}+\gamma d=x^{\#} .
$$

Combining the above with Proposition 2, one obtains the result below. Let $X^{\gamma}$ denote the set of minimizers of (5).

Proposition 3 If $X^{\gamma}$ is a singleton and $s^{\gamma}\left(x^{\gamma}\right)$ remains constant for $\gamma \in\left(0, \gamma^{*}\right]$, and then

$$
x^{\gamma}+\gamma d=x^{0}
$$

where $d$ solves

$$
\left(A^{T} A+W^{\gamma}\left(x^{\gamma}\right)\right) d=s^{\gamma}\left(x^{\gamma}\right),
$$


and $x^{0}$ solves $(2)$.

Proof If $X^{\gamma}$ is a singleton for sufficiently small $\gamma>0$ then the $\left(\begin{array}{c}A \\ W^{\gamma}\left(x^{\gamma}\right)\end{array}\right)$ has full rank. Then using the sign constancy, the result follows from the previous analysis, Corollary 1 and Proposition 2.

Proposition 3 implies that if for $\gamma>0$ sufficiently small one has uniqueness of the minimizer and the sign constancy condition satisfied then one can zoom along to a solution of problem (2) from a solution of (5) after solving a system of linear equations. Note that we used SVD only for the sake of analysis. In actual computation one does not need to compute the SVD. A more economical factorization of $\left(\begin{array}{c}A \\ W^{\gamma}\left(x^{\gamma}\right)\end{array}\right)$ (e.g., $\left.Q R\right)$ can be used.

Before exploring further the property of sign constancy and ties between the solutions to (2), (5) and a sparse solution, we shall first weaken the uniqueness condition on $x^{\gamma}$ in Proposition 3. To do this we need to study the dual problem to (5). First, let us observe that the dual problem to (5) is the following strictly concave quadratic optimization problem (the dual can be obtained using Lagrange duality similarly to the development in Madsen et al. 1998):

$$
\max _{\xi}\left\{-\frac{\gamma}{2} \xi^{T}\left(I_{m}+A A^{T}\right) \xi+b^{T} \xi:\left\|A^{T} \xi\right\|_{\infty} \leq 1\right\}
$$

with a unique solution, $\xi^{\gamma}$. Optimality conditions for the pair of primal-dual problems imply the following relations between a solution $x^{\gamma}$ to (5) and the dual solution $\xi^{\gamma}$ :

$$
\begin{gathered}
A^{T} \xi^{\gamma}=\frac{1}{\gamma} W^{\gamma}\left(x^{\gamma}\right) x^{\gamma}+s^{\gamma}\left(x^{\gamma}\right), \\
\frac{1}{\gamma} \xi^{\gamma}=b-A x^{\gamma} .
\end{gathered}
$$

Furthermore, by a classical result of Mangasarian and Meyer (1979), for sufficiently small $\gamma>0$, we have that $\xi^{\gamma}=\xi^{*}$ is the least weighted-norm solution of the dual problem (3) using the norm term $\left\|\left(\begin{array}{c}I \\ A^{T}\end{array}\right) x\right\|_{2}$ after viewing the dual problem above as

$$
\max _{\xi}\left\{-\frac{\gamma}{2}\left\|\left(\begin{array}{c}
I \\
A^{T}
\end{array}\right) \xi\right\|_{2}^{2}+b^{T} \xi:\left\|A^{T} \xi\right\|_{\infty} \leq 1\right\}
$$

i.e., $\xi^{*}$ solves the problem

$$
\min _{\xi \in \Xi^{0}}\left\|\left(\begin{array}{c}
I \\
A^{T}
\end{array}\right) \xi\right\|_{2}
$$


Remark 1 The aforementioned property has an important implication for algorithm development. Namely, even if there is no way to compute a priori the critical value of $\gamma$ when extrapolation will take us to an optimal solution of (2), we can check complementary slackness conditions (4) at the points $x^{0}$ and $\xi^{\gamma}$. If they are satisfied, we stop with an optimal primal-dual pair, otherwise we continue with a smaller $\gamma$ value. This remark is valid for all extrapolation results of the subsequent sections.

\section{Extreme points and extrapolation}

Now recall that $x$ is an extreme point of $X^{\gamma}$ if and only if $x=\lambda y+(1-\lambda) z$ implies $x=y=z$ for any $\lambda \in(0,1)$.

Lemma $3 x$ is an extreme point of $X^{\gamma}$ if and only if $\left(\begin{array}{c}A \\ W^{\gamma}(x)\end{array}\right)$ has full rank.

Proof We proceed as in Li and Swetits (1998). Suppose that $x \in X^{\gamma}$ and $\left(\begin{array}{c}A \\ W^{\gamma}(x)\end{array}\right)$ has rank less than $n$. Then there exists a vector $u \in \mathbb{R}^{n}$, not identically zero, such that $\left(\begin{array}{c}A \\ W^{\gamma}(x)\end{array}\right) u=0$. Now we can pick an $\epsilon>0$ sufficiently small such that $(x \pm \epsilon u)_{i}>\gamma$ for all $i$ such that $x_{i}>\gamma$ and $(x \pm \epsilon u)_{i}<-\gamma$ for all $i$ such that $x_{i}<-\gamma$. Observe that $A(x \pm \epsilon u)=A x$ and $W^{\gamma}(x)(x \pm \epsilon u)=W^{\gamma}(x) x$. Thus, we have $s^{\gamma}(x \pm \epsilon u)=s^{\gamma}(x)$. Hence, both $x \pm \epsilon u$ satisfy (11) and we get that both $x \pm \epsilon u \in X^{\gamma}$. Since $x=\frac{1}{2}(x+\epsilon u)+\frac{1}{2}(x-\epsilon u)$ with $u \neq 0, x$ is not an extreme point of $X^{\gamma}$.

Suppose $x \in X^{\gamma}$ and $\left(\begin{array}{c}A \\ W^{\gamma}(x)\end{array}\right)$ has rank $n$. If $y, z \in X^{\gamma}$ are such that $x=\lambda y+(1-\lambda) z$ for some $\lambda \in(0,1)$ then by (17) we have $\gamma\left(A^{T} \xi^{\gamma}\right)_{i}=x_{i}$ if and only if $y_{i}=z_{i}=\gamma\left(A^{T} \xi^{\gamma}\right)_{i}$. Thus we have $W^{\gamma}(x) x=W^{\gamma}(x) y=W^{\gamma}(x) z$. By (18) we have $\gamma(b-A x)_{i}=\xi_{i}^{\gamma}$ if and only if $\gamma(b-A y)_{i}=\xi_{i}^{\gamma}=\gamma(b-A z)_{i}$, which implies $A x=A y=A z$. Hence we have $\left(\begin{array}{c}A \\ W^{\gamma}(x)\end{array}\right) x=\left(\begin{array}{c}A \\ W^{\gamma}(x)\end{array}\right) y=\left(\begin{array}{c}A \\ W^{\gamma}(x)\end{array}\right) z$, which in turn implies that $x=y=z$. Therefore, $x$ is an extreme point of $X^{\gamma}$.

Remark 2 The proof of the above lemma points out to an algorithmic procedure to construct an extreme point optimal solution departing from an optimal point $x^{\gamma}$ where $\left(\begin{array}{c}A \\ W^{\gamma}\left(x^{\gamma}\right)\end{array}\right)$ is rank deficient. It suffices to find a non-trivial null-space vector, move along this vector to increase rank (i.e., the number of components with absolute value within $\gamma$ ) and repeat this step until one ends up with an extreme point optimal solution, which is guaranteed to occur after a finite number of steps.

Using the above lemma and the analysis leading to Proposition 3, we can state directly the extrapolation property even in the absence of uniqueness of the minimizer, provided the sign vector associated with an extreme point minimizer remains constant for sufficiently small $\gamma$. 
Proposition 4 Let $x^{\gamma}$ be an extreme point minimizer of (5) for $\gamma \in\left(0, \gamma^{*}\right]$. If $s^{\gamma}\left(x^{\gamma}\right)$ remains constant for $\gamma \in\left(0, \gamma^{*}\right]$, then

$$
x^{\gamma}+\gamma d=x^{0}
$$

where d solves

$$
\left(A^{T} A+W^{\gamma}\left(x^{\gamma}\right)\right) d=s^{\gamma}\left(x^{\gamma}\right)
$$

and $x^{0}$ solves $(2)$.

It turns out that we can weaken the conditions used in the analysis of the present section even further.

Lemma 4 For every instance of the problem there exists a sign vector $s \in\{0, \pm 1\}^{n}$ where for all $\gamma^{u}>0$ there exists a $\gamma \in\left(0, \gamma^{u}\right]$ and an extreme point minimizer of (5), $x^{\gamma}$, such that $s^{\gamma}\left(x^{\gamma}\right)=s$.

Proof Assume that the claim is false. Given $S=\{0, \pm 1\}^{n}$, for every $s \in S$ there will be a $\gamma_{s}>0$ such that for all $\gamma \in\left(0, \gamma_{s}\right]$ and for every extreme point minimizer $x^{\gamma}$, we have $s^{\gamma}\left(x^{\gamma}\right) \neq s$. For all $\gamma \in\left(0, \min _{s \in S} \gamma_{s}\right.$, there exists a minimizer extreme point, $x^{\gamma}$, of (5) (since the solution set is non-empty and compact for all $\gamma>0$ ) and $s^{\gamma}\left(x^{\gamma}\right) \in S$, which is a contradiction.

Now, we relax Proposition 3 as follows.

Proposition 5 For sufficiently small $\gamma^{*}$ for every $\gamma \leq \gamma^{*}$ any extreme point of $X^{\gamma}$ will satisfy

$$
x^{\gamma}+\gamma d=x^{0}
$$

where d solves

$$
\left(A^{T} A+W^{\gamma}\left(x^{\gamma}\right)\right) d=s^{\gamma}\left(x^{\gamma}\right)
$$

and $x^{0}$ solves (2).

Proof For every extreme point of $X^{\gamma}$ the corresponding $\left(\begin{array}{c}A \\ W^{\gamma}\left(x^{\gamma}\right)\end{array}\right)$ has full rank (by Lemma 3). Let $\hat{S}$ be the set of vectors $s \in\{0, \pm 1\}^{n}$, for which there exists a $\gamma_{s}>0$ such that for all $\gamma \in\left(0, \gamma_{s}\right]$ and for every extreme point $x^{\gamma}$ of $X^{\gamma}$, we have $s^{\gamma}\left(x^{\gamma}\right) \neq s$ (Lemma 4 implies $\{0, \pm 1\}^{n} \backslash \hat{S} \neq \emptyset$ ). Let $\gamma^{*}=\min _{s \in \hat{S}} \gamma_{s}$ and for any given $\gamma \in\left(0, \gamma^{*}\right]$, let $x^{\gamma}$ be an extreme point of $X^{\gamma}$. Then for any $\gamma \in\left(0, \gamma^{*}\right]$, there exists a $\gamma^{\prime} \in(0, \gamma)$ and a corresponding extreme point minimizer $x^{\gamma^{\prime}}$ such that $s^{\gamma}\left(x^{\gamma}\right)=s^{\gamma^{\prime}}\left(x^{\gamma^{\prime}}\right)$. Then for every $\gamma<\gamma^{*}$ and any extreme point $x^{\gamma}$ of $X^{\gamma}$, we can find a sequence of extreme point minimizers $x^{\gamma^{k}}$ such that $\gamma^{k} \rightarrow 0$ and $s^{\gamma^{k}}\left(x^{\gamma^{k}}\right)=s^{\gamma}\left(x^{\gamma}\right)$. 
Therefore, $\gamma=\gamma^{k}$ satisfies sign constancy in (15), combined with $\left(\begin{array}{c}A \\ W^{\gamma^{k}}\left(x^{\gamma^{k}}\right)\end{array}\right)=\left(\begin{array}{c}A \\ W^{\gamma}\left(x^{\gamma}\right)\end{array}\right)$ being full rank (Lemma 3) this completes the proof.

As a consequence of Proposition 5, even if the minimizer of (5) is not unique one can use any extreme point of the solution set for sufficiently small $\gamma>0$ to reach a solution of problem (2).

\section{Sign constancy and ties among solutions}

Now, we shall turn to the question of sign constancy and ties among various solutions.

Definition 1 A dual optimal solution $\xi^{*}$ to (3) is called non-degenerate if there exists a primal optimal solution $x^{*}$ such that the following conditions hold:

$$
\begin{aligned}
& \left(A^{T} \xi^{*}\right)_{i}=1 \Longrightarrow x_{i}^{*}>0, \\
& \left(A^{T} \xi^{*}\right)_{i}=-1 \Longrightarrow x_{i}^{*}<0, \\
& \left|\left(A^{T} \xi^{*}\right)_{i}\right|<1 \Longrightarrow x_{i}^{*}=0 .
\end{aligned}
$$

For any sign vector $s$, we define $\kappa_{s}^{+}=\left\{i: s_{i}=1\right\}$, and $\kappa_{s}^{-}=\left\{i: s_{i}=-1\right\}$ with $\kappa_{s}=\kappa_{s}^{+} \cup \kappa_{s}^{-}$, and $\kappa_{s}^{0}=\left\{i: s_{i}=0\right\}$.

Proposition 6 Let $x_{0}$ be a unique solution to (2) with $\mathbf{s}_{0}=s^{0}\left(x_{0}\right)$ and $x^{\gamma}$ be the unique minimizer of (5) with dual solution $\xi^{\gamma}$ satisfying non-degeneracy conditions (1) (with $\left.x_{0}\right)$ for sufficiently small $\gamma>0$. Then, there exists $\gamma^{*}$ such that $s^{\gamma}\left(x^{\gamma}\right)$ remains constant for $\gamma \in\left(0, \gamma^{*}\right]$.

Proof Since for sufficiently small $\gamma>0$ the unique $x^{\gamma} \rightarrow x_{0}$ by Proposition 2, we have $\left|x_{i}^{\gamma}\right|>\gamma$ for $i \in \kappa_{\mathbf{s}_{0}}$ and sufficiently small $\gamma>0$. Because $\xi^{\gamma} \in \Xi^{0}$ for sufficiently small $\gamma>0$ and by the non-degeneracy assumption, we have from (17) that $\left|x_{i}^{\gamma}\right| / \gamma<1$ for $i \in \kappa_{\mathbf{s}_{0}}^{0}$ and $\gamma$ sufficiently small.

For the next result, we use $s^{0} \in\{0, \pm 1\}^{n}$ defined before with the diagonal matrix $W^{0}($.$) derived from s^{0}($.$) using W_{i i}^{0}=1-\left(s_{i}^{0}\right)^{2}$ for $i=1, \ldots, n$, as before.

Proposition 7 Let $x_{0}$ be a (sparse) solution of $A x=b$ with $s=s^{0}\left(x_{0}\right)$ and $W=W^{0}\left(x_{0}\right)$. If there exists a solution $d$ to the system of linear equations:

$$
\left(A^{T} A+W\right) d=s
$$

with the property

$$
\|W d\|_{\infty} \leq 1
$$

then there exists $\gamma^{*}$ such that 


$$
s^{\gamma}\left(x_{0}-\gamma d\right)=s
$$

and $x_{0}-\gamma d$ solves (5) for $\gamma \in\left(0, \gamma^{*}\right]$. Furthermore, $x_{0}$ solves (2).

Proof We can re-write (19) as

$$
\left(A^{T} A+W\right) d=\frac{1}{\gamma}\left(A^{T} A+W\right) x_{0}-\frac{1}{\gamma} A^{T} b+s
$$

for some $\gamma>0$ since $A x_{0}=b$ and $W x_{0}=0$. By simple algebra, rearranging the previous we obtain

$$
\left(A^{T} A+W\right)\left(x_{0}-\gamma d\right)=A^{T} b-\gamma s
$$

Now, let $\delta=\min \left\{\left|\left(x_{0}\right)_{i}\right|:\left|\left(x_{0}\right)_{i}\right| \neq 0\right\}$. Choose $0<\gamma_{2}<\delta$ such that for $\gamma \in\left(0, \gamma_{2}\right]$ one has

$$
\begin{aligned}
& \left(x_{0}-\gamma d\right)_{i}>\gamma_{2} \text { for } i \in \kappa_{s}^{+}, \\
& \left(x_{0}-\gamma d\right)_{i}<-\gamma_{2} \text { for } i \in \kappa_{s}^{-} .
\end{aligned}
$$

Combining the above with (20), we have that $s^{\gamma}\left(x_{0}-\gamma d\right)=s$, and using (21) $x_{0}-\gamma d$ solves (5) for $\gamma \in\left(0, \gamma^{*}\right]$ where $\gamma^{*}=\gamma_{2}$.

On the other hand, the fact that there exists a solution $d$ to (19) with $\|W d\|_{\infty} \leq 1$ implies that one can take $\lambda \equiv A d$. Hence, one has $A^{T} \lambda=-W d+s$. But $\|W d\|_{\infty}=\|-W d\|_{\infty}$. Hence $x_{0}$ solves (2) by Proposition 1.

If $x_{0}$ of the previous result also satisfies a mild regularity condition in addition to the one already presented, then an immediate corollary is obtained as follows.

Corollary 2 Let $x_{0}$ be a (sparse) solution of $A x=b$ with $s=s^{0}\left(x_{0}\right)$ and $W=W^{0}\left(x_{0}\right)$ such that $\left(\begin{array}{c}A \\ W\end{array}\right)$ has full rank. If the unique solution $d$ to the system of linear equations:

$$
\left(A^{T} A+W\right) d=s
$$

has the property

$$
\|W d\|_{\infty} \leq 1
$$

then there exists $\gamma^{*}$ such that

$$
s^{\gamma}\left(x_{0}-\gamma d\right)=s
$$

and $x_{0}-\gamma d$ solves (5) for $\gamma \in\left(0, \gamma^{*}\right]$. Furthermore, $x_{0}$ solves (2).

In particular, the above two results (Proposition 5 and 2) are valid for a sparse solution to the linear system of equations. Hence, they constitute sufficient conditions for the sparsest solution to be a minimizer of (2). 
A more substantial sign constancy result is proved below relaxing some of the conditions imposed previously (i.e., non-degeneracy) in the present section.

Theorem 1 Let $x_{0}$ be a unique solution to (2) with $s_{0}=s^{0}\left(x_{0}\right)$. If $x^{\gamma}$ solving (5) is unique for sufficiently small $\gamma$ then there exists $\gamma^{*}$ such that $s^{\gamma}\left(x^{\gamma}\right)$ remains constant for $\gamma \in\left(0, \gamma^{*}\right]$ with $\kappa_{s_{0}}^{+} \subseteq \kappa_{s^{\gamma}}^{+}, \kappa_{s_{0}}^{-} \subseteq \kappa_{s^{\gamma}}^{-}$.

Proof Let $x_{0}$ be the unique solution to (2) with $\mathbf{s}_{0}=s^{0}\left(x_{0}\right)$. Since the number of different sign vectors $s$ is finite, there must a exist a sequence of positive numbers $\gamma_{1}, \gamma_{2}, \ldots$, with $\gamma_{k} \searrow 0$ for $k \rightarrow \infty$ such that $s^{\gamma}\left(x^{\gamma}\right)$ is constant for $\gamma=\gamma_{k}$ for $k=1,2, \ldots$. Denote this sign vector by s. Let $\mathcal{D}=\left\{i:\left|\left(A^{T} \xi^{\gamma}\right)_{i}\right|=1\right\} \cap \kappa_{\mathbf{s}_{0}}^{0}$, and $\mathcal{S}=\left\{s \in S: s_{i}=\mathbf{s}_{0 i}\right.$ for $\left.i \notin \mathcal{D}\right\}$. Since $x^{\gamma} \rightarrow x_{0}$ by Proposition 2, we have $\left|x_{i}^{\gamma}\right|>\gamma$ for $i \in \kappa_{\mathbf{s}_{0}}$ and sufficiently small $\gamma>0$. Since $\xi^{\gamma} \in \Xi^{0}$ for sufficiently small $\gamma>0$, we have from (17) that $\left|x_{i}^{\gamma}\right| / \gamma<1$ for $i \in \kappa_{\mathbf{s}_{0}}^{0} \backslash \mathcal{D}$ and $\gamma$ sufficiently small. Therefore, we must have that $\mathbf{s} \in \mathcal{S}$ since $\gamma_{k} \searrow 0$.

Now, consider again $\mathbf{s}$ (the sign vector that is encountered infinitely many times as defined above) and its associated diagonal matrix $\mathbf{W}$. Let the SVD factorization of $\left(\begin{array}{c}A \\ \mathbf{W}\end{array}\right)=\mathbf{U} \boldsymbol{\Sigma} \mathbf{V}^{T}$ and consider the system

$$
\left(A^{T} A+\mathbf{W}\right) d=\mathbf{s} .
$$

This system is consistent by Lemma 2 since $x_{\gamma}$ satisfies (12). We have, as previously,

$$
d_{\gamma}=\sum_{j=1}^{n} \frac{\beta_{j}}{\sigma_{j}^{2}} \mathbf{v}_{j}
$$

if $\left(\begin{array}{c}A \\ \mathbf{W}\end{array}\right)$ has full rank. Since $\psi_{i}(\gamma)=-\gamma\left(d_{\gamma}\right)_{i}$ is a rational function of $\gamma$, it can only have a finite number of oscillations as $\gamma \searrow 0$; hence there exists $\gamma_{1}^{*}>0$ such that either $\left|\psi_{i}(\gamma)\right|>1$ for $\gamma \in\left(0, \gamma_{1}^{*}\right]$ or $\left|\psi_{i}(\gamma)\right| \leq 1 \gamma \in\left(0, \gamma_{1}^{*}\right]$. If $i \notin \kappa_{\mathbf{s}_{0}}^{0}$ then $\left(x_{0}\right)_{i}=0$ and $\left(x_{0}\right)_{i}-\gamma\left(d_{\gamma}\right)_{i}=\psi_{i}(\gamma)$. Hence, the $i$ th component of $s^{\gamma}\left(x_{0}-\gamma d_{\gamma}\right)$ is constant for $\gamma \in\left(0, \gamma_{1}^{*}\right]$. Since $d_{\gamma}$ is bounded, the other components of $s^{\gamma}\left(x_{0}-\gamma d_{\gamma}\right)$ must also be constant in some interval $\left(0, \gamma_{2}^{*}\right]$. Therefore, $s^{\gamma}\left(x_{0}-\gamma d_{\gamma}\right)$ is constant for $\gamma \in\left(0, \gamma_{3}^{*}\right]$ where $\gamma_{3}^{*}=\min \left\{\gamma_{1}^{*}, \gamma_{2}^{*}\right\}$.

Now, let $\gamma=\gamma_{k} \in\left(0, \gamma_{3}^{*}\right]$ be a value for which $s^{\gamma}\left(x^{\gamma}\right)=\mathbf{s}$. It follows from the above development that the unique minimizer $x^{\gamma}$ is given as $x_{0}-\gamma d$.

We can now merge the results of Proposition 3 with Theorem 1 into the following.

Theorem 2 Let $x_{0}$ be a unique solution to (2) with $s_{0}=s^{0}\left(x_{0}\right)$. If $x^{\gamma}$ solving (5) is unique for sufficiently small $\gamma$, then

$$
x^{\gamma}+\gamma d=x_{0},
$$

where $d$ solves 


$$
\left(A^{T} A+W^{\gamma}\left(x^{\gamma}\right)\right) d=s^{\gamma}\left(x^{\gamma}\right) .
$$

We have made the assumption that both the solution $x_{0}$ to (2) and a minimizer $x^{\gamma}$, for $\gamma>0$ sufficiently small, to (5) are unique. A legitimate question is whether the uniqueness of $x_{0}$ implies that of $x^{\gamma}$. The answer is negative as the following example shows.

\section{Example 1 Let}

$$
A=\left(\begin{array}{cccc}
1 & -1 & 1 / 2 & -1 \\
0 & 0 & -17 & 2
\end{array}\right)
$$

with $b=\left(\begin{array}{ll}-1 & 2\end{array}\right)^{T}$. The unique solution to (2) is $x^{0}=\left(\begin{array}{llll}0 & 0 & 0 & 1\end{array}\right)^{T}$. For sufficiently small $\gamma \in(0,0.195]$, the set of minimizers of (5) is the interval between the extreme points

$$
(-\gamma 2 \gamma-\gamma / 21-17 \gamma / 4)^{T}
$$

and

$$
(-2 \gamma \gamma-\gamma / 21-17 \gamma / 4)^{T}
$$

We can weaken the uniqueness condition of Theorem 1 as shown in the next result.

Proposition 8 Let $x_{0}$ be a unique solution to (2) with $s=s^{0}\left(x_{0}\right)$ and $W=W^{0}\left(x_{0}\right)$ and let $\bar{s}$ (and $\bar{W}$ derived from $\bar{s}$ ) be a sign vector such that $\kappa_{\bar{s}}^{0} \subseteq \kappa_{s}^{0}$ with $\kappa_{s}^{+} \subseteq \kappa_{\bar{s}}^{+}$, and $\kappa_{s}^{-} \subseteq \kappa_{\bar{s}}^{-}$with $\left(\begin{array}{c}A \\ \bar{W}\end{array}\right)$ having full rank. If the unique solution $d^{*}$ to

$$
\left(A^{T} A+\bar{W}\right) d=\bar{s}
$$

has the properties $\left\|\bar{W} d^{*}\right\|_{\infty} \leq 1$, and for all $i$ such that $s_{i}=0$ and $\bar{s}_{i} \neq 0$ it holds that $\bar{s}_{i} d_{i}^{*}<0$ and $\left|d_{i}^{*}\right|>\max _{j: s_{j}=\bar{s}_{j}=0}\left|d_{j}^{*}\right|$ then there exists $\gamma^{*}$ such that

$$
s^{\gamma}\left(x_{0}-\gamma d\right)=\bar{s}
$$

and $x_{0}-\gamma d$ is an extreme point minimizer of (5) for $\gamma \in\left(0, \gamma^{*}\right]$.

Proof Similar to the proof of Proposition 7, we re-write (25) as

$$
\left(A^{T} A+\bar{W}\right) d^{*}=\frac{1}{\gamma}\left(A^{T} A+\bar{W}\right) x_{0}-\frac{1}{\gamma} A^{T} b+\bar{s}
$$

for some $\gamma>0$ since $A x_{0}=b$ and $\bar{W} x_{0}=0$. Rearranging the previous we obtain

$$
\left(A^{T} A+\bar{W}\right)\left(x_{0}-\gamma d^{*}\right)=A^{T} b-\gamma \bar{s} .
$$

Now, let $\delta=\min \left\{\left(x_{0}\right)_{i}:\left|\left(x_{0}\right)_{i}\right| \neq 0\right\}$. Choose $0<\gamma_{2}<\delta$ such that for $\gamma \in\left(0, \gamma_{2}\right]$ one has 


$$
\begin{aligned}
& \left(x_{0}-\gamma d^{*}\right)_{i}>\gamma_{2} \text { for } i \in \kappa_{\bar{s}}^{+}, \\
& \left(x_{0}-\gamma d^{*}\right)_{i}<-\gamma_{2} \text { for } i \in \kappa_{\bar{s}}^{-},
\end{aligned}
$$

due to the conditions imposed on $d^{*}$. Combining the above with (20), we have that $s^{\gamma}\left(x_{0}-\gamma d\right)=\bar{s}$, and using (26) $x_{0}-\gamma d$ solves (5) for $\gamma \in\left(0, \gamma^{*}\right]$ where $\gamma^{*}=\gamma_{2}$, and is an extreme point minimizer.

Example 2 Notice that all conditions of the previous proposition are fulfilled in Example 1. If one takes $\bar{s}=\left(\begin{array}{llll}0 & 1 & 0 & 1\end{array}\right)^{T}$ then one gets $d^{*}=\left(\begin{array}{llll}1 & -2 & 1 / 2 & 17 / 4\end{array}\right)$, and for $\gamma$ small enough, one obtains the extreme point minimizers $\left(\begin{array}{llll}-\gamma & 2 \gamma-\gamma / 2 & 1-17 \gamma / 4\end{array}\right)^{T}$. On the other hand, if one takes $\bar{s}=\left(\begin{array}{llll}-1 & 0 & 0 & 1\end{array}\right)^{T}$, one gets the $d^{*}=(2-11 / 217 / 4)$ and recovers the extreme points $(-2 \gamma \gamma-\gamma / 21-17 \gamma / 4)^{T}$ for sufficiently small $\gamma$.

To conclude the paper, we give a necessary condition for recovery of a sparsest solution; a similar result is also given in Pinar (2019) for exact recovery of an individual sparse vector. The necessary condition gives an unexpected relationship between the sparsest solution recovery (by recovery it is meant that the sparsest solution should be unique in (2)) and the unique solution to (16) being the unique solution to (3) as well.

Theorem 3 Let $\hat{x}$ be a sparsest solution to $A x=b$ with $\|\hat{x}\|_{0}<m$. If $\hat{x}$ solves (2) uniquely then for sufficiently small $\gamma>0$ the unique solution to (16) is not the unique solution to (3).

Proof For sufficiently small $\gamma>0$ the unique solution to (16) is the least norm solution of (3). By the theory of linear programming (c.f. Bertsimas and Tsiksiklis 1997), the dual problem (3) admits an extreme point optimal solution $\xi^{*}$ with the set $\mathcal{A}\left(\xi^{*}\right)=\left\{i:\left|\left(A^{T} \xi^{*}\right)_{i}\right|=1\right\}$ having at least $m$ elements. If $\xi^{*}$ is the unique optimal solution to (3) [(it is the unique optimal solution to (16)] then by the GoldmanTucker strict complementarity theorem (c.f. Williams 1970) and the uniqueness of $\hat{x}$, the support of $\hat{x}$ should have cardinality at least $m$, which is a contradiction.

The algorithmic implications of the results of the paper, as well as efficient gradient-based algorithms for solving the problem (5) (especially for larger instances) are the subject of on-going research, c.f., (Kizılkale et al. 2020), and will be discussed in a subsequent work.

\section{References}

Aybat NS, Iyengar G (2011) A first-order smoothed penalty method for compressed sensing. SIAM J Optim 21(1):287-313

Aybat NS, Iyengar G (2012) A first-order augmented lagrangian method for compressed sensing. SIAM J Optim 22(2):429-459 
Bertsimas D, Tsiksiklis J (1997) Introduction to Linear Optimization, Belmont. Athena Scientific, Massachusetts

Bryan K, Leise T (2013) Making do with less: an introduction to compressed sensing. SIAM Rev 55:547-566

Candès EJ, Tao T (2006) Decoding by linear programming. IEEE Trans Inform Theory 52:4203-4215

Chen BT, Madsen K, Zhang Sh (2005) On the characterization of quadratic splines. J Optim Theory Appl 124(1):93-111

Cheng W, Dai Y-H (2018) Gradient based method with active set strategy for $\ell_{1}$ optimization. Math Comput 87(311):1283-1305

Cheng W, Dai Y-H An active set Newton-CG method for $\ell_{1}$ optimization, Applied and Computational Harmonic Analysis, to appear

Cheng W, Hu QJ, Li D (2019) A fast conjugate gradient algorithm with active set prediction for $\ell_{1}$ optimization. Optim Methods Softw 34(6):1277-1305

Cheng W, Chen Z, Li D (2015) Non-monotone spectral gradient method for sparse recovery. Inv Prob Imag 9(3):815-833

Cheng W, Li D (2018) A preconditioned conjugate gradient method with active set strategy for $\ell_{1}$ -regularized least squares. J Oper Res Soc China 6:571-585

Chrétien S (2010) An alternating $\ell_{1}$ approach to the compressed sensing problem. IEEE Sig Proc Lett 17:181-184

Chen S, Donoho D, Saunders M (1998) Atomic decomposition by basis pursuit. SIAM J Sci Comput 20:33-61

Donoho DL (2005) Compressed sensing. IEEE Trans Inform Theory 51:1289-1306

Donoho DL (2006) For most large underdetermined systems of linear equations the minimal $\ell_{1}$-norm solution is also the sparsest solution. Comm Pure Appl Math 59:797-829

Elad M (2010) Sparse and redundant representations: from theory to applications in signal and image processing. Springer, New York

Foucart S, Rauhut H (2013) A Mathematical Introduction to Compressive Sensing. Springer, New York

Fuchs JJ (2004) On sparse representations in arbitrary redundant bases. IEEE Trans Inform Theor 50(6):1341-1344

Huber PJ (1981) Robust Statistics. Wiley, New York

Kızılkale C, Chandrasekaran S, Ç. Pınar M, Gu M (2020) Gradient based adaptive restart is linearly convergent, Technical Report, http://www.ie.bilkent.edu.tr/ mustafap/pubs/Restartnew4.pdf. Accessed July 2020

Lanza A, Morigi S, Selesnick IW, Sgallari F (2019) Sparsity-inducing non-convex, non-separable regularization for convex image processing. SIAM J Imag Sci 12(2):1099-1134

Lee JD, Sun Y, Saunders MA (2014) Proximal Newton-type methods for minimizing composite functions. SIAM J Optim 24(3):1420-1443

Li W, Swetits JJ (1998) The linear $\ell_{1}$ estimator and the Huber M-estimator. SIAM J Optim 8:457-475

Madsen K, Nielsen HB (1993) A finite smoothing algorithm for linear $\ell_{1}$ estimation. SIAM J Optim 3:223-235

Madsen K, Nielsen HB, Pınar MÇ (1994) New characterizations of $\ell_{1}$ solutions of overdetermined systems of linear equations. Oper Res Lett 16:159-166

Madsen K, Nielsen HB, Pınar MÇ (1998) A finite continuation algorithm for bound constrained quadratic programming. SIAM J Optim 9:62-83

Mangasarian OL, Meyer RR (1979) Nonlinear perturbations of linear programs. SIAM J Control Optim 17:745-752

Nesterov Yu (2004) Introductory lectures on convex optimization: a basic course. Kluwer Academic Publishers, Norwell, MA

Nesterov Yu (2005) Smooth minimization of nonsmooth functions. Math Program 103:127-152

Pınar MÇ (2019) Necessary and sufficient conditions for noiseless sparse recovery via convex quadratic splines. SIAM J Matrix Anal Appl 40(1):194-209

Selesnick I (2017) Sparse regularization via convex analysis. IEEE Trans Sig Process 65(17):4481-4494

Singaraju D, Tron R, Elhamifar E, Yang AY, Sastry SS (2012) On the lagrangian biduality of sparsity minimization, ICASSP 2012, IEEE Proceedings, 3429-3432

Wang S, Chen X, Dai W, Selesnick IW, Cai G (2018) Vector minimax concave penalty for sparse representation. Dig Sig Process 83:165-179 
Wang J, Zhang F, Huang J, Wang W, Yuan C (2019) A non-convex penalty function with integral convolution approximation for compressed sensing. Sig Process 158:116-128

Wen Z, Yin W, Goldfarb D, Zhang Y (2010) A fast algorithm for sparse reconstruction based on shrinkage, subspace optimization, and continuation. SIAM J Sci Comp 32(4):1832-1857

Williams AC (1970) Complementarity theorems for linear programming. SIAM Rev 12:135-137

Publisher's Note Springer Nature remains neutral with regard to jurisdictional claims in published maps and institutional affiliations. 